\title{
SUICIDALITY: COLLEGE STUDENTS' ATTITUDES AND BEHAVIOURS IN MALAYSIA
}

\author{
Meg Milligan* \\ Department of Psychology, Troy University, Riverfront Campus 402-B, \\ Phenix City, AL 36867, United States \\ E-mail: mmilligan@troy.edu \\ See Hoon Peow** \\ Chief Executive Officer Berjaya University College, Level 11 West, \\ Berjaya Times Square, No 1 Jalan Imbi, 55100 Kuala Lumpur, Malaysia \\ E-mail: hpsee@berjaya.edu.my
}

\section{Hall P. Beck***}

Department of Psychology, Appalachian State University, 215 Smith Wright Hall, 222 Joyce Lawrence LN, ASU Box 32108, Boone, NC 28608, United States

E-mail: beckhp@appstate.edu

\section{Sherrionda Crawford $* * * *$}

Counseling, Rehabilitation, and Interpreter Training Division, Troy University, 368 Hawkins Hall, Troy, AL 36082, United States

E-mail: scrawford@troy.edu

Kanessa Miller Doss *****

Department of Psychology, Troy University, 231 Montgomery Street, Room 300, Montgomery, AL 36104, United States

E-mail:kmdoss@troy.edu

Published online: 25 January 2022

To cite this article: Milligan, M., See, H. P., Beck, H. P., Crawford, S. and Doss, K. M. 2022. Suicidality: College students' attitudes and behaviours in Malaysia. International Journal of Asia Pacific Studies 18 (1): 151-167. https://doi. org/10.21315/ijaps2022.18.1.7

To link to this article: https://doi.org/10.21315/ijaps2022.18.1.7 


\begin{abstract}
The World Health Organization (WHO) identifies suicidality, the experience of suicidal thoughts or behaviour including attempts, as an ongoing global public health concern. The WHO estimated that more than 79\% of suicides in 2016 occurred in low to middle income nations, with 60\% of these occurring in Asia. Suicides are often underreported and misclassified as death by other causes. As a result, obtaining accurate information is difficult. This problem is exacerbated in developing countries facing limited budgets, legislative barriers, and social stigma. These realities place greater responsibility on researchers to collect and interpret data. This study addressed this issue in a sample of 176 college students in Malaysia. The Suicide Behaviours Questionnaire-Revised (SBQ-R), which examines individual attitudes and behaviour concerning suicide, was administered and demographic information was collected on gender, age, ethnicity, and religion. Data were analysed using Statistical Package for the Social Sciences (SPSS, version 25). Results showed no significant gender effects, but there were significant differences across religions. This is discussed in terms of buffering effects and minority-majority status. This study increases knowledge about suicidality among college students in Malaysia, a high-risk group. Recommendations for prevention and intervention and future research are included.
\end{abstract}

Keywords: SBQ-R, suicidality, suicidal ideation, suicidal behaviour, college students, Malaysia

\title{
INTRODUCTION
}

Increased media attention highlights the significance of suicidality for Malaysian adolescents and young adults. On 13 May 2019, a 16-year-old girl in Malaysia chose death by suicide after her Instagram poll results said, two to one, that she should die rather than live (Fullerton 2019). In January 2018, Malaysians were shocked to read about the widely reported and highly controversial death of the 13-year-old girl student who was accused of stealing her teacher's iPhone. After being accused and questioned, she was found hanging in her room and unconscious; she was later declared dead in the hospital. This case has since gone to court (Basyir 2018). In April 2018, an outstanding student jumped off a building in Seremban, a town near Kuala Lumpur, Malaysia. It is believed that she succumbed to depression due the stress to excel in her studies, despite scoring 10 As in Sijil Pelajaran Malaysia or SPM (Malaysian Certificate of Education; a public examination at the end of higher secondary school) (Fong 2018). In June 2018, a college 
student shot himself in his college toilet with a pistol stolen from the shooting club of which he was a member. It is believed that he had been suffering from depression (Kwan 2018). In October 2018, a 15-year-old student killed herself a day before she was due to sit for the Pentaksiran Tingkatan Tiga or PT3 examination (Form 3 Assessment; a public examination for Form 3 students) (Tan 2018). These cases sparked wide discussion about suicide in Malaysia, and it is believed that there may be many more unreported suicides or attempts by Malaysian students for various reasons.

Research data concerning Malaysian students' suicidality and the media attention these cases and others have received illustrate the need for further investigation into student suicide in Malaysia. From available reports, "in Malaysia, there has been an average of 56 deaths a year caused by 'intentional self-harm', based on data from Health Ministry hospitals nationwide between 2012 and 2016. In 2016, there were 54 deaths - a decrease from the 67 deaths in 2015 and 61 in 2014" (Yuen 2018). According to a survey conducted by the Ministry of Health Malaysia, " $10 \%$ of secondary school students have thought about committing suicide before while $7.3 \%$ of the students actually planned to commit suicide" (Fun 2018). The Movement Control Order (MCO) during the COVID-19 pandemic in 2020 has exacerbated the situation. It was reported that suicide attempts spiked during the MCO (Benjamin and Devi 2020). A total of 500 suicide attempts were reported in 2020 (Bernama 2020). According to the National Health and Mobility Survey 2019 published in $2020,2.3 \%$ or about half a million Malaysians are depressed, and 424,000 children are found to have mental health problems (Institute for Public Health 2020).

The World Health Organization (WHO 2016) reports that over 800,000 individuals a year die by suicide worldwide. This number of reported suicides equates to one person every 40 seconds. Death by suicide is the second leading cause of death among 15 to 29 -year-olds. Many people in this age range are college students who often are burdened with school-life balance, and sometimes additional stressors such as work and family pressures to succeed academically. Furthermore, the WHO (2018) estimated that more than 79\% of suicides in 2016 occurred in low to middle income nations, $60 \%$ of which were in Asia (O'Connor and Pirkis 2016). While Southeast Asia accounts for $25.9 \%$ of the global population, the overall suicide rate in Southeast Asian countries accounts for $39.1 \%$ of global deaths by suicide (WHO 2016). Specific to Malaysia, The Malaysian Psychiatric Association (2007) reported a $60 \%$ rise in suicide rates over the preceding 45 years. The WHO (2013) implemented a Mental Health Action Plan that included targeting a $10 \%$ 
reduction in suicides worldwide by 2020 . This data has not been published yet, but reaching that target seems less likely due to the COVID-19 pandemic's associated increases in suicide (Banerjee et al. 2021).

There is a myth that questioning an individual about suicidality increases suicide risk. Suicidality is the experience of suicidal thoughts (ideation) or behaviour, and includes suicide attempts (parasuicides) and suicides (Jager-Hyman et al. 2017). Although one might expect that broaching this topic would engender anxious feelings, research does not support the notion that inquiring about suicidality increases suicidal tendencies. Gould et al. (2005) investigated the impact of students responding to research questions about suicidal thoughts and found no adverse effects. Dazzi et al. (2014) also discovered that there were no published studies with a statistically significant increase in suicidal ideation among individuals questioned about suicidal thoughts. These authors recommended that engaging in dialogue about suicide may reduce ideation which may influence the individual to increase help-seeking behaviours.

Suicides are underreported and often misclassified as deaths by other causes or other violent death (OVD), and more accurate information is needed to understand this global public health problem (O'Connor and Pirkis 2016; Shrivastava et al. 2015). Obtaining accurate data is a challenge around the world. Some of the reasons for this are similar, such as disinterest, stigma, and insufficient knowledge and professional training, while others vary by location (O'Connor and Pirkis 2016). For example, the unreliability of data estimates in Malaysia is compounded by a lack of national data collection (WHO 2018), no data from some of the states, religious beliefs (Ab Razak 2017), and legal sanctions against attempted suicide (e.g., Section 309 of the Malaysian Penal Code). That dearth places greater responsibility on other sources of data, such as research studies. The primary purpose of the current study is to add empirical data to help understand suicidal attitudes and behaviour among college students in Malaysia and to identify associated demographics.

\section{RESEARCH IN MALAYSIA}

Sinniah et al. (2014) searched databases from 1845 to 2012 using the search term "suicide and Malaysia", and sought local sources of information as well. They identified 38 studies in English (published 1969 to 2011) that investigated suicide attempts (parasuicides), and found that $76 \%$ of those were 
descriptive (i.e., demographics, mental disorders, method, and causes). Their recommendations for further research included validating Western measures for Malaysian populations, and investigating effects of gender, ethnicity, and other cultural variables.

Armitage et al. (2015) synthesised findings from 39 research studies related to suicide and self-harm in Malaysia. They noted that rates reported by the National Suicide Registry Malaysia (NSRM) only included deaths that were medically certified, thereby overlooking half of all deaths. Furthermore, the cause of many deaths was "indeterminate" or accidental. Many of these might have been suicides. Maniam Thambu reported the parasuicide to suicide rate in Malaysia as ten to one (Mustaffa et al. 2014).

Meanwhile, Armitage et al. (2015) summarised that, overall, males, particularly ages $15-44$, were at greater risk to die by suicide, and women, primarily ages 14-40, were more likely to self-harm; lower socioeconomic status was also a significant risk factor. Males and individuals 40 years old and younger accounted for $67 \%-76 \%$ of suicides. Suicidal ideation past year prevalence rates were $6 \%-8 \%$, similar to the rate found in the 2012 Malaysia Global School-Based Student Health Survey (GHSH).

The GHSH remains the most comprehensive study of 13 to 17-year-old secondary school students' health in Malaysia (Low and Binns 2014; Yusoff et al. 2014). This study used a nationally representative sample. Ahmad et al. (2014: 2) reported that $7.9 \%$ of the 25,174 students who were surveyed reported suicidal ideation, defined as "ever seriously considered attempting suicide in the past 12 months". Positive correlates included female gender, Indian and Chinese ethnicity, anxiety, depression, being bullied, substance use, stress, and family violence. These can be considered risk factors for suicidality among this age group in Malaysia, while having a close friend at school was identified as a protective factor.

The authors observed that these results were probably underestimates, considering stigma and religious dogma. For example, Islam, the major religion in Malaysia, and some forms of Christianity, most notably Catholicism, prohibit suicide. Furthermore, Islam requires burial within 24 hours, which can hinder accurately specifying cause of death. In contrast, the Hindu religion, more commonly practiced by people with Indian ancestry, sanctions some types of suicidal behaviour, which may contribute to the higher observed rates.

Kok et al. (2011) studied a sample of secondary and post-secondary students and identified three stressful precipitators, namely, romantic relationships, family, and school. Chinese students rated academics as the 
primary motivator leading to suicide. They discussed the stresses from a transitional society with weakening cultural and family ties as significant issues.

Armitage et al. (2015) found no consistently identified most common method used for death by suicide in Malaysia. Ethnic differences were noted in Kuala Lumpur in a 1999 study, namely, hanging by Chinese, jumping by Malays, and poisoning by Indians. Regional differences were notable. Poisoning, especially by pesticide ingestion, was most common in rural areas (most likely due to easy access), while hanging and jumping were more likely in urban locations (an unintended consequence of urbanisation). It is conjectured that risk of jumping increases as cities build more and higher buildings and may account for this rural-urban difference in methods used. Married Indian women were at higher risk of suicide and self-harm compared to other groups.

Suicidality is a major concern in Malaysia, as evidenced by the establishment of the Healthy Mind Programme in schools, suicide hotlines, and designating September as National Suicide Prevention Awareness Month. The NSRM Annual Report 2009, the most recently published, listed the youngest person to die by suicide as 14 years old and the oldest as 94 , while the report in 2008 recorded the youngest as 12 . The youths who died by suicide were most likely to be males of Indian ethnicity, with hanging the primary method followed by poisoning. Contrary to other studies, "mental and physical illnesses, family history of mental illness, and previous suicide attempt were surprisingly not common among the suicide completers" (Bahar et al. 2015: 1). The 2009 report stated that "suicide is a rare phenomenon with a high impact... [and] a worthwhile focus of investigation" (foreword).

A study using a random sample of 65 college students in Malaysia (Mustaffa et al. 2014) reported that suicidal ideation was more common among male college students compared to female college students. Syed Saddiq Syed Abdul Rahman, Youth and Sports Minister of Malaysia, said "I am genuinely worried about the state of our youth's mental health...It's a national issue which must be taken seriously" (Fullerton 2019). In that spirit, this study provides data concerning attitudes and behaviours related to suicide or thoughts of suicide among college students in Malaysia. Clearly, more research is needed to address the many unanswered questions in the suicidality literature pertaining to Malaysia. This study investigates some of these questions. Most importantly, it identifies demographic factors associated with higher risk for suicidality (operationally defined by scores on the Suicide Behaviours Questionnaire-Revised, SBQ-R) in our sample of college students in Malaysia and discusses explanations for these findings. 


\section{RESEARCH METHOD}

\section{Participants}

One hundred and ninety-nine undergraduates enrolled in a Malaysian university with multiple campuses were asked to participate. Four students elected not to do so. Sixteen participants responded to fewer than three of the four SBQ-R items and were not included in the following analysis. In addition, three students were identified as multivariate outliers using procedures recommended by Tabachnick and Fidell (2007) and were excluded from further analyses.

The ethnicities of the remaining 176 students were: African American/ Black, 1; American Indian, 1; Asian, 3; Chinese, 75; Hispanic, 1; Indian, 70; Malay, 21; Pacific Islander, 1; and 3 respondents reported their ethnicity as "Other". The gender identifications of the sample were: female, 119; male, 45; gender variant/nonconforming, 2; transgender female, 4; transgender male, 5; and 1 student preferred not to answer. Regarding religious identification, the demographics were Buddhism, 41; Chinese Religion, 10; Christianity, 22; Hinduism, 62; Islam, 27; Taoism, 4, and 10 students responded "Other". The ages of the students were: $18-20$ years, $41 ; 21-23$ years, 77 ; 24-29 years, 55; and 30 years or older, 3 .

\section{Instrument}

The SBQ- $\mathrm{R}$ is a 4-item questionnaire that examines four dimensions of suicidality: (1) lifetime suicidal ideations and/or suicide attempts, (2) frequency of suicidal ideations over the past 12 months, (3) threat of suicide, and (4) likelihood of suicidal behaviour in the future (Osman et al. 2001). The SBQ-R has been used in a study of university students in China (Lew et al. 2020) and has been used in research in Malaysia (Abu Talib and Abdollahi 2017).

The SBQ-R's purpose is to identify at-risk individuals and specific risk behaviours. The SBQ-R uses Likert type scoring, such as never (1 point) to very often (5 points). The total score on the SBQ-R is summative, with a range from 3 to 18. For adults in the general population, a score of 7 or higher is considered at risk of suicide. For adults in a psychiatric inpatient programme, a score of 8 or more is considered at risk for suicide (Osman et al. 2001). 
This study uses an alternative but equivalent method of calculating mean scores. The lowest possible score using the standard summative approach is 3 but there are 4 items. Thus, the lowest mean is 0.75 . Mean item scores rather than total scores are used for this reason. Moreover, only persons who responded to 3 or more are used in this study. However, some persons responded to 3 and not all 4 items, and we do not want to further reduce the sample size. Therefore, we computed a mean based on the number of items answered.

\section{Procedure}

After Institutional Review Board (IRB) approval, a recruitment letter was sent via email to students enrolled in a university in Malaysia. The email recruitment letter included the informed consent document. To consent to participation in the study, respondents were provided a link at the bottom of the email that also granted access to the SBQ-R (Osman et al. 2001). Demographic information collected consisted of age, gender, ethnicity, and religion, since suicidality research has identified these variables as salient. Contact information for suicide prevention hotlines and local crisis resources were provided regardless of participation. This information was furnished within the informed consent process and at the completion of the survey. Incentives or motivators were not offered for participation.

Qualtrics web-based survey software was used for data collection. All participants were treated in accord with the American Psychological Association's Ethical Principles of Psychologists and Code of Conduct (American Psychological Association 2002). Email addresses were not captured to maintain the anonymity of the participants' data.

\section{RESULTS}

All statistical tests used IBM ${ }^{\circledR}$ SPSS $^{\circledR}$ Statistics 25 software. Twenty three of 176 students elected not to answer the first SBQ-R question, "Have you ever thought about or attempted to kill yourself?". In contrast, only six students did not answer one of the remaining SBQ-R items. Therefore, two measures of internal consistency were calculated, one for students who answered all four items $(N=153$, Cronbach alpha $=0.82)$ and one for students who responded to the last three questions $(N=170$, Cronbach alpha $=0.78)$. Reliability was determined to be satisfactory, especially given that the questionnaire consisted of only four items. 
We used analysis of variance (ANOVA), "statistical tests for comparing the means of two or more groups" (Corty 2014: G1) to identify any significant results in our data, specifically, which group characteristics were more predictive of suicidality as measured by the SBQ-R. Had the sample been larger we would have performed a two-factor ANOVA with gender and religion serving as predictor variables. However, this would have left some cells with too few participants to conduct a reliable analysis. Therefore, we performed five separate one-way ANOVAs for each predictor in which Mean Suicide Scores and the scores on the four items of the SBQ-R served as criterion measures. Given the number of statistical tests, alpha was set at 0.01 .

Due to their small number, for statistical purposes, the 11 respondents identifying themselves as gender variant/nonconforming, transgender female, or transgender male were grouped into a single category called "Other". None of the five one-way ANOVAs using gender as the predictor approached statistical significance, all $p>0.10$. Thus, we found no support for the proposition that gender identification was related to scores on the SBQ-R.

Table 1 contains the Ms, SDs and results of the five one-way ANOVAs with religion as the predictor variable. The pattern of results found in mean SBQ-R scores was repeated on each of the four items. Christians reported the highest mean scores followed by students in the Other category. Respondents professing to be practitioners of Buddhism, Chinese religion, and Islam tended to obtain scores approximating the sample mean. On all items, Hindus reported the lowest scores.

Table 1: Relationship of professed religion to SBQ-R survey scores

\begin{tabular}{llllllllll}
\hline Item & Buddhism & $\begin{array}{l}\text { Chinese } \\
\text { religion }\end{array}$ & Christian & Hinduism & Islam & Other & $\begin{array}{l}\text { Sample M } \\
\text { and SD }\end{array}$ & $\begin{array}{l}\mathrm{F} \\
(5,170)\end{array}$ & $\begin{array}{l}\text { Partial } \\
\eta^{2}\end{array}$ \\
\hline $\begin{array}{l}\text { Mean } \\
\text { SBQ-R }\end{array}$ & 1.21 & 1.41 & 2.23 & 1.07 & 1.41 & 1.84 & 1.38 & $10.77^{*}$ & 0.24 \\
Score & & $(0.69)$ & $(1.06)$ & $(0.35)$ & $(0.90)$ & $(0.89)$ & $(0.79)$ & & \\
1 & 1.35 & 1.75 & 2.38 & 1.15 & 1.57 & 2.30 & 1.50 & $8.98^{*}$ & 0.23 \\
& $(0.63)$ & $(1.04)$ & $(1.20)$ & $(0.49)$ & $(0.90)$ & $(1.25)$ & $(0.88)$ & & \\
2 & 1.46 & 1.70 & 2.42 & 1.16 & 1.70 & 2.21 & 1.57 & $10.66^{*}$ & $0.24 \eta$ \\
& $(0.75)$ & $(0.68)$ & $(1.08)$ & $(0.41)$ & $(0.99)$ & $(1.05)$ & $(0.86)$ & & \\
3 & 1.15 & 1.40 & 1.91 & 1.10 & 1.44 & 1.57 & 1.32 & $9.43^{*}$ & 0.23 \\
& $(0.48)$ & $(0.52)$ & $(0.68)$ & $(0.30)$ & $(0.70)$ & $(0.76)$ & $(0.59)$ & & \\
4 & 0.85 & 0.70 & 1.95 & 0.85 & 0.81 & 1.29 & 1.01 & $3.40^{*}$ & 0.09 \\
& $(1.32)$ & $(1.25)$ & $(1.89)$ & $(0.57)$ & $(1.38)$ & $(1.38)$ & $(1.26)$ & & \\
\hline
\end{tabular}

Notes: All values not in parentheses are Ms. SDs are in parentheses. ${ }^{*} p<0.01$.

Items

1. Have you ever thought about or attempted to kill yourself?

2. How often have you thought about killing yourself in the past year?

3. Have you ever told someone that you were going to commit suicide, or that you might do it?

4. How likely is it that you will attempt suicide someday? 
Individuals reporting a total summative score of 7 or greater $(M=>$ 1.75 per item) on the SBQ-R are at significant risk (high risk) for suicidal behaviours. In the total sample, 48 of $176(27 \%)$ of students yielded mean scores of at least 1.75. The breakdown by religion (at-risk, total students of a given religion) was: Buddhism 8 of 41 (20\%), Chinese religion 3 of $10(30 \%)$, Christianity 15 of $22(68 \%)$, Hinduism 5 of $62(8 \%)$, Islam 9 of $27(33 \%)$, and Taoism plus Other 8 of 14 (57\%) students reported mean summative scores equal or greater than 1.75. A chi-square test of independence yielded a statistically significant relationship between a student's religion and whether they were a significant risk for suicidal behaviours, $\chi^{2}(5, N=176)=38.18$, $p<0.001$.

A reexamination of the data resulted in an interesting and unexpected finding. The percentage of at-risk students was strongly related to the frequency of various religious groups within our sample. For the purpose of analysis, the three religious groups with the lowest frequencies (Chinese religion, Christianity, and Other religion) were labelled "minority religions" and the three groups with the highest frequencies (Buddhism, Hinduism, and Islam) were labelled "majority religions". Twenty six of the 46 minority religion students $(57 \%)$ were at-risk. In contrast, only 22 of $130(17 \%)$ majority religion students were at-risk. The difference in the likelihood of minority and majority religion students being at-risk was statistically significant, $\chi^{2}(1, N=176)=26.86, p<0.001$.

\section{CONCLUSION}

The purpose of the current study is to add to empirical understanding of attitudes and behaviours related to suicide or thoughts of suicide among college students in Malaysia and to identify demographic risk factors. Acknowledging the complexity and multi-determined nature of suicidal behaviours, this study has examined associations between suicidality and ethnicity, gender, religion, and age, and has found that religion is the prominent variable. Clearly, Christians are at highest risk. Hindus are at lowest risk, which seems contrary to other findings that Indians are at higher risk, but it is possible that religion (Hinduism) and ethnicity (Indian) are confounding rather than conflicting. Hindus are not a minority at this institution and that may provide buffers to suicidality, perhaps through social support and enhanced self-esteem.

There is a significant positive correlation between ethnicity and overall suicidality. However, while all the Malays are Muslims and 53 out of 62 Indians are Hindus, the Chinese students are distributed across four 
religions, namely, Buddhism, Christianity, Chinese religion, and Taoism, with correspondingly different levels of risk. There exists an ethnicity with religion interaction among the Chinese group that is not as strongly apparent within the other ethnicities.

A striking observation from our results is that our data suggest a strong relationship between a religion's majority/minority status in Malaysia and suicide risk, such that minorities seem to be at greater risk. Risk is not distributed equally. If we look at the data more closely, those who are represented less frequently in our data are at higher risk overall. Chinese religion (10, 10 Chinese), Taoism/Other (14, 9 Chinese), and Christianity (22, 17 Chinese) are the religious categories least represented in our sample, and $56 \%$ of these individuals are in the high-risk group, while Islam (27), Buddhist (41), and Hindu (62) have greater representation and only $17 \%$ are considered high risk. This is a huge difference. It is notable that $36(78 \%)$ out of the 46 individuals who are least represented identified as Chinese. Since 40 out of the 41 Buddhists are Chinese as well, it is possible that their religious identification has an effect on their risk of suicidality, or there might be interactions with social support and purpose in life (Wang et al. 2016). Future research could investigate this possibility. It is worth noting a tendency among Chinese in Malaysia to misreport their religion as Buddhism, although most Malaysian Chinese are actually followers of a syncretised religion normally referred to as Chinese Religion (Tan 1983). Lately this has improved, with Buddhist groups and Taoist groups trying to clarify things. Future studies are advised to be aware of this study's results as well as possible changes in religious identification. Possible explanations for these findings include the following:

1. These observed differences are not differences in suicidality, but rather are differences in willingness to disclose.

2. Some religions either encourage and/or do not discourage suicidality.

3. It is nothing about the religion itself, but it is about the greater culture these religions tend to be embedded in. It is something about the broader culture reflecting ethnicity. For example, Kok et al. (2011) have found that Chinese students rate academics as their primary stressor. Our sample is a student population with a high degree of family pressure to succeed, and Chinese students may feel this pressure more acutely compared to others. These pressures combined with minority religion may cause higher suicide risk, and 
4. It is not so much the specific doctrine of the religion or the culture, but it is about being a minority in the college or in the broader culture.

These observations and possible explanations warrant further investigation.

The small sample size is a limitation in this study. However, the demographic distributions appear to represent this college student population, and these students are from multiple campus locations, which is a strength. It is notable that this study was conducted before the COVID-19 pandemic, and may underestimate current suicidality, while providing data for comparative purposes with a post-pandemic study. Therefore, recommendations for future study include a post-COVID-19 replication study with a larger, representative sample, as well as studies across tertiary schools in Malaysia.

The results indicate unacceptable levels of suicidal ideation and behaviour, which may reflect high stress levels, inadequate coping skills, and untreated mental disorders among college student populations in Malaysia. Moderating effects of religion are also indicated, which can be helpful to many college students.

It is also noteworthy that suicidality is closely connected to substance use and co-occurring mental health disorders (Chen et al. 2005; May et al. 2012). Future measures should assess substance use and mental health history. In addition, there are cultural differences in causal attributions. For example, it is common for Southeast Asians to believe that supernatural forces are the aetiology of their mental health concerns (Pang et al. 2018). Research indicates that one's mental health literacy influences help-seeking behaviour (Eisenberg et al. 2009; van der Ham 2011). Therefore, another path for future research is to investigate specific perceptions and knowledge related to mental illness and help-seeking behaviours among college students in Malaysia.

The Malaysian Mental Health Association (MMHA) is an example of an organisation that is changing perceptions and disseminating accurate information. The MMHA is aware of the dire need to address suicidality and is equally committed to developing outreach programmes aimed at mental wellness and destigmatising mental health services. Accordingly, they have collaborated with WHO to develop and establish public education platforms and programmes to improve knowledge of various mental illnesses and reduce suicidality. Platforms such as Mind Hub, implemented on 5 October 2019, and Great Minds address the increase in suicide, as well as educate the public on statistics and coping skills. This partnership will be instrumental as it relates to increasing mental health literacy, destigmatising mental illness, and increasing help-seeking behaviours which could decrease future suicide rates. 
But a better understanding of the suicidal tendency is needed as well. Our study contributes to this understanding and has important implications for suicide prevention and intervention among college students. There is a need to address barriers to treatment and address the impact of factors such as religion and stigma related to mental health. Additionally, knowing the demographics of the high-risk groups helps mental health experts identify these individuals and develop targeted interventions for them.

\section{NOTES}

* Dr Meg Milligan is an associate professor in the Department of Psychology at Troy University, Alabama, U.S. She teaches in the U.S. and Malaysia and conducts study abroad programmes, most notably to Costa Rica. She has presented at numerous national and international conferences, including invited keynote addresses. Her academic background includes a BA in Sociology and Anthropology from the American University of Beirut, Lebanon and a $\mathrm{PhD}$ in Counselling Psychology from Auburn University, Alabama, U.S. Dr Milligan completed an internship at the Veteran's Administration Medical Center in Tuskegee, Alabama, U.S. with rotations in neuropsychology, outpatient posttraumatic stress disorder, geriatrics, and inpatient psychiatry, and is a licensed psychologist in Alabama. She serves on the Board of Directors of the Association for Distance Education and Independent Learning. Dr Milligan's current research interests include suicide, the history of psychology, and narcissism.

** Dr Hall P. Beck holds a PhD in Social Psychology from the University of North Carolina at Greensboro, North Carolina, U.S. For 34 years, Dr Beck happily professed at Appalachian State University in Boone, North Carolina, U.S. Following his retirement in 2018, Dr Beck was appointed Professor Emeritus. He no longer lectures but maintains an active research programme. Dr Beck has published over 70 articles including publications in some of the world's most prestigious journals, most notably in the areas of human computer interaction, college student retention, and the history of psychology.

*** Dr See Hoon Peow holds a PhD in Sociology and Anthropology from International Islamic University Malaysia, Master of Philosophy in Sociology from University of Malaya and LLB (Hons) from University of Glamorgan (now University of South Wales). He has an extensive work experience in the education industry with exposure in both academic and business operations. He is currently Chief Executive Officer of BERJAYA University College. Prior to joining Berjaya University College, he has held top management positions in other educational institutions. He has wide academic interest in humanities and social sciences. Although he is a full-time manager, he is active in research and publishing. He has published many books and papers locally and internationally with reputable publishers and journals. He has also presented papers in local and international conferences. 
**** Dr Sherrionda Crawford, PhD, LPC-S, NCC, ACS is an Associate Professor of School and Clinical Mental Health Counselling in the Division of Counseling, Rehabilitation and Interpreter Training at Troy University (Phenix City, AL). She is credentialed as both a Licensed Professional Counsellor (Alabama and Georgia) and a Certified School Counsellor in the State of Alabama. Dr Crawford has extensive experience as a clinician with both adolescents and adults. Dr Crawford actively volunteers for the National Board of Certified Counselors (NBCC) as a scholarship reviewer and mentor. Her research interests include trends in school counselling to include interpersonal violence and suicide prevention, as well as skill acquisition for counsellors-in-training.

${ }^{* * * * *}$ Dr Kanessa Miller Doss is an Associate Professor of Psychology at Troy UniversityMontgomery Campus. She is a Nationally Certified School Psychologist (NCSP) and National Certified Counselor (NCC). Dr Doss is also a certified school counsellor and school psychologist in the state of Alabama. She has nine years of experience as a publicschool biological sciences educator and five years as a school psychologist consultant. As an advocate for suicide prevention, Dr Doss became a certified Question, Persuade, Refer (QPR) Gatekeeper in Suicide Prevention Training instructor. She is a conference co-founder of the Alabama Higher Education Suicide Prevention Conference. She is a member and board member of several professional organisations. Dr Doss actively serves the community as a board member for Christian Services for Children of Alabama (CSCA) and Renewed Economic Development. Her research interests are social-emotional functioning with an emphasis on school/college campus violence, bullying, bullycide, youth and young adult suicide, school psychology advocacy, and accessibility issues.

\section{REFERENCES}

Ab Razak, A. 2017. Cultural construction of psychiatric illness in Malaysia. Malaysian Journal of Medical Sciences 24 (2): 1-5. https://doi.org/10.21315/mjms2017.24.2.1

Abu Talib, M. and Abdollahi, A. 2017. Spirituality moderates hopelessness, depression, and suicidal behavior among Malaysian adolescents. Journal of Religion and Health 56 (3): 784-795. https://doi.org/10.1007/s10943-015-0133-3

Ahmad, N., Cheong, S. M., Ibrahim, N. and Rosman, A. 2014. Suicidal ideation among Malaysian adolescents. Asia Pacific Journal of Public Health 26 (5): 1-7. https:// doi.org/10.1177/1010539514540746

American Psychological Association. 2002. Ethical principles of psychologists and code of conduct. American Psychologist 57 (12): 1060-1073. https://doi.org/10.1037/0003066X.57.12.1060

Armitage, C. J., Panagioti, M., Rahim, W. A., Rowe, R. and O'Connor, R. C. 2015. Completed suicides and self-harm in Malaysia: A systematic review. General Hospital Psychiatry 37 (2): 153-165. https://doi.org/10.1016/j.genhosppsych.2014.12.002

Bahar, N., Ismail, W. S. W., Hussain, N., Haniff, J., Bujang, M. A., Hamid, A. M., Yusuff, Y., Nordin, N. and Ali, N. H. 2015. Suicide among youth in Malaysia: What do we know? Asia-Pacific Psychiatry 7 (2): 223-229. https://doi.org/10.1111/appy.12162 
Banerjee, D., Kosagisharaf, J. R. and Sathyanarayana Rao, T. S. 2021. 'The dual pandemic' of suicide and COVID-19: A biopsychosocial narrative of risks and prevention. Psychiatry Research 295: 113577. https://doi.org/10.1016/j.psychres.2020.113577

Basyir, M. 2018. Death of Penang girl accused of iPhone theft ignites outrage. New Straits Times, 1 February 2018. https://www.nst.com.my/news/nation/2018/02/330953/ death-penang-girl-accused-iphone-theft-ignites-outrage (accessed 17 March 2021).

Benjamin, N. and Devi, V. 2020. Suicides spike during MCO. The Star, 11 August 2020. https://www.thestar.com.my/news/nation/2020/08/11/suicides-spike-during-mco (accessed 17 March 2021).

Bernama. 2020. Almost 500,000 Msians depressed; nearly 500 suicide attempts this year. New Straits Times, 10 October 2020. https://www.nst.com.my/news/ nation/2020/10/631154/almost-500000-msians-depressed-nearly-500-suicideattempts-year (accessed 17 March 2021).

Chen, P. C. Y., Lee, L. K., Wong, K. C. and Kaur, J. 2005. Factors relating to adolescent suicidal behavior: A cross-sectional Malaysian school sample. Journal of Adolescent Health 37 (4): 337.e11-337.e16. https://doi.org/10.1016/j.jadohealth.2004.10.018

Corty, E. W. 2014. Using and interpreting statistics. New York: Worth Publishers.

Dazzi, T., Gribble, R., Wessely, S. and Fear, N. T. 2014. Does asking about suicide and related behaviours induce suicidal ideation? What is the evidence? Psychological Medicine 44 (16): 3361-3363. https://doi.org/10.1017/S0033291714001299

Eisenberg, D., Downs, M. F., Golberstein, E. and Zivin, K. 2009. Stigma and help seeking for mental health among college students. Medical Care Research and Review 66 (5): 522-541. https://doi.org/10.1177/1077558709335173

Fong, F. 2018. Depressed student allegedly commits suicide in Seremban, cites academic stress. New Straits Times, 4 April 2018. https://www.nst.com.my/news/crimecourts/2018/04/352814/depressed-student-allegedly-commits-suicide-serembancites-academic (accessed 17 March 2021).

Fullerton, J. 2019. Teenage girl kills herself 'after Instagram Poll' in Malaysia. The Guardian, 15 May 2019. https://www.theguardian.com/world/2019/may/15/ teenage-girl-kills-herself-after-instagram-poll-in-malaysia (accessed 17 March 2021).

Fun, P. (2018). MOH: 10\% of high school students in M'sia have thought of committing suicide. World of Buzz, 10 October 2018. https://worldofbuzz.com/moh-10-ofhigh-school-students-in-msia-have-thought-of-committing-suicide/ (accessed 17 March 2021).

Gould, M. S., Marrocco, F. A., Kleinman, M., Thomas, J. G., Mostkoff, K., Cote, J. and Davies, M. 2005. Evaluating iatrogenic risk of youth suicide screening programs: A randomized controlled trial. Journal of the American Medical Association 293 (13): 1635-1643. https://doi.org/10.1001/jama.293.13.1635

Institute for Public Health. 2020. National Health and Morbidity Survey (NHMS) 2019 (NMRR-18-3085-44207), non-communicable diseases, healthcare demand, and health literacy: Key findings. National Institutes of Health (NIH) Ministry of Health Malaysia. http://iptk.moh.gov.my/images/technical report/2020/ 4_Infographic_Booklet_NHMS_2019_-_English.pdf (accessed 14 February 2021). 
Jager-Hyman, S., Wenzel, A. and Brown, G. K. 2017. Suicidality, treatment of. In The SAGE encyclopedia of abnormal and clinical psychology, ed. Wenzel, A. Thousand Oaks, CA: SAGE Publications.

Kok, J. K., Gan, C. C. and Goh, L. Y. 2011. Youths talk about death: Causes for suicide. International Journal of Social Science and Humanity 1 (2): 145-149. https://doi. org/10.7763/IJSSH.2011.V1.25

Kwan, L. 2018. Subang Jaya student commits suicide by shooting himself in college toilet. World of Buzz, 27 June 2018. https://www.worldofbuzz.com/subang-jaya-studentcommits-suicide-by-shooting-himself-in-college-toilet/ (accessed 20 December 2020).

Lew, B., Chistopolskaya, K., Osman, A., Huen, J. M. Y., Abu Talib, M. and Lueng, A. N. M. 2020. Meaning in life as a protective factor against suicidal tendencies in Chinese University students. BMC Psychiatry 20: 1-9. https://doi.org/10.1186/ s12888-020-02485-4

Low, W.-Y. and Binns, C. 2014. Global school-based student health survey, Malaysia. Asia Pacific Journal of Public Health 26 (5): 7S-8S. https://doi. org/10.1177/1010539514545287

Malaysian Psychiatric Association. 2007. Suicide - it's an SOS! http://www.psychiatrymalaysia.org/article.php?aid=504 (accessed 14 February 2021).

May, A. M., Konsky, E. D. and Klein, D. N. 2012. Predicting future suicide attempts among depressed suicide ideators: A 10-year longitudinal study. Journal of Psychiatric Research 46 (7): 946-952. https://doi.org/10.1016/j.jpsychires.2012.04.009

Mustaffa, S., Aziz, R., Mahmood, M. N. and Shuib, S. 2014. Depression and suicidal ideation among university students. Procedia - Social and Behavioral Sciences 116: 4205-4208. https://doi.org/10.1016/j.sbspro.2014.01.917

O'Connor, R. C. and Pirkis, J., eds. 2016. The international handbook of suicide prevention (2nd ed.). Hoboken, NJ: Wiley-Blackwell.

Osman, A., Bagge, C. L., Gutierrez, P. M., Konick, L. C., Kopper, B. A. and Barrios, F. X. 2001. The Suicidal Behaviors Questionnaire-Revised (SBQ-R): Validation with clinical and nonclinical samples. Assessment 8 (4): 443-454. https://doi. org/10.1177/107319110100800409

Pang, S., Subramaniam, M., Lee, S. P., Lau, W. Y., Abdin, E., Chua, B. Y., Picco, L., Vaingankar, J. A. and Chong, S. A. 2018. The Singaporean public beliefs about the cause of mental illness: Results from a multi-ethnic population-based study. Epidemiology and Psychiatric Sciences 27 (4): 403-412. https://doi.org/10.1017/ S2045796017000105

Shrivastava, S. R., Shrivastava, P. S. and Ramasamy, J. 2015. Public health strategies to ensure reduction in incidence in middle and low income nations. Journal of Neuroscience in Rural Practice 6 (4): 619-621. https://doi.org/10.4103/09763147.165431

Sinniah, A., Maniam, T., Oei, T. P. and Subramaniam, P. 2014. Suicide attempts in Malaysia from the year 1969 to 2011. The Scientific World Journal 718367. https:// doi.org/10.1155/2014/718367

Tabachnick, B. G. and Fidell, L. S. 2007. Using multivariate statistics (5th ed.). Boston, MA: Pearson Education Inc. 
Tan, C.-B. 1983. Chinese religion in Malaysia: A general view. Asian Folklore Studies 42 (2): 217-252. https://doi.org/10.2307/1178483

Tan, S. C. 2018. Student found dead on day of PT3 Exam. The Star, 2 October 2018. https://www.thestar.com.my/news/nation/2018/10/02/student-found-dead-on-dayof-pt3-exam/ (accessed 17 March 2021).

van der Ham, L., Wright, P., Van, T. V., Doan, V. D. K. and Broerse, J. E. W. 2011. Perceptions of mental health and help-seeking behavior in an urban community in Vietnam: An explorative study. Community Mental Health Journal 47 (5): 574582. https://doi.org/10.1007/s10597-011-9393-x

Wang, Z., Koenig, H. G., Ma, H. and Al Shohaib, S. 2016. Religion, purpose in life, social support, and psychological distress in Chinese university students. Journal of Religion and Health 55: 1055-1064. https://doi.org/10.1007/s10943-016-0184-0

World Health Organization (WHO). 2018. Malaysia: Risk of premature death due to NCDs. World Health Organization Noncommunicable Diseases (NCD) Country Profiles, 2018. https://www.who.int/nmh/countries/mys_en.pdf (accessed 14 February 2021).

. 2016. WHO suicide fact sheet. http://www.who.int/mediacentre/factsheets/fs398/en/ (accessed 14 February 2021).

. 2013. Comprehensive mental health action plan 2013-2020. http://apps.who.int/gb/ ebwha/pdf_files/WHA66/A66_R8-en.pdf?ua=1 (accessed 14 February 2021).

Yuen, M. 2018. Looking for the light. The Star, 1 July 2018. https://www.thestar.com. $\mathrm{my} / \mathrm{news} /$ nation/2018/07/01/looking-for-the-light-mental-health-is-on-thegovernments-radar-with-the-health-ministry-working-on/ (accessed 21 January 2021).

Yusoff, F., Saari, R., Naidu, B. M., Ahmad, N. A. Omar, A. and Aris, T. 2014. Methodology of the National School-Based Health Survey in Malaysia, 2012. Asia Pacific Journal of Public Health 26 (5): 9S-17S. https://doi.org/10.1177/1010539514542424 